\title{
Attitudes and Beliefs of Physical Therapist and Physical Therapist Assistant Program Directors in the United States Towards Interprofessional Education
}

\author{
Lawrence Ramiscal \\ Augusta University, Iramiscal.pt@gmail.com \\ Christopher Truelove Jr. \\ Augusta University, CTruelove@augusta.edu \\ Vahe Heboyan \\ Augusta University, vheboyan@augusta.edu \\ Gianluca De Leo \\ Augusta University, gdeleo@augusta.edu
}

Follow this and additional works at: https://nsuworks.nova.edu/ijahsp

Part of the Interprofessional Education Commons, Physical Therapy Commons, and the Physiotherapy Commons

\section{Recommended Citation}

Ramiscal L, Truelove C, Heboyan V, De Leo G. Attitudes and Beliefs of Physical Therapist and Physical Therapist Assistant Program Directors in the United States Towards Interprofessional Education. The Internet Journal of Allied Health Sciences and Practice. 2022 Jan 03;20(1), Article 13.

This Manuscript is brought to you for free and open access by the College of Health Care Sciences at NSUWorks. It has been accepted for inclusion in Internet Journal of Allied Health Sciences and Practice by an authorized editor of NSUWorks. For more information, please contact nsuworks@nova.edu. 


\title{
Attitudes and Beliefs of Physical Therapist and Physical Therapist Assistant Program Directors in the United States Towards Interprofessional Education
}

\begin{abstract}
Purpose: To investigate the attitudes and beliefs of physical therapy (PT) and physical therapist assistant (PTA) program directors towards interprofessional education (IPE). We hypothesized that Communication and Ethics would be the most important competencies among program directors. Methods: A crosssectional survey based on previously utilized instruments modified for the profession of PT was sent to PT and PTA program directors. One hundred sixteen responses were analyzed using frequency analysis for demographic data and non-parametric Mann-Whitney $U$ t-test for group differences. Results: While the majority of program directors agree that IPE is important, with Communication as the most important IPE competency, most PTA program directors do not support the importance of accreditation in implementing IPE (pConclusion:Program directors agree that IPE is vital to student learning, with Communication as the most important IPE competency. However, specific differences between PT and PTA program directors emerged primarily on IPE implementation, the role of accreditation, resource support, and resource utilization.
\end{abstract}

\section{Author Bio(s)}

Lawrence S. Ramiscal, PT, DPT, Ph.D., is an Assistant Professor at the Department of Physical Therapy, Augusta University in Augusta, GA. He is also a board-certified orthopedic physical therapy specialist (OCS) and Fellow of the American Academy of Orthopaedic Manual Physical Therapists (FAAOMPT).

Christopher A. Truelove, Jr., MSCIN, RRT-NPS, is an Assistant Professor and Respiratory Therapy Program Director at the Department of Undergraduate Health Professions, Augusta University in Augusta, GA. He is a practicing respiratory therapist and a neonatal/pediatric specialist.

Vahé Heboyan, Ph.D., is an Associate Professor at the Department of Population Health Science, Augusta University in Augusta, GA.

Gianluca de Leo, Ph.D., MBA, is a Professor at the Department of Interdisciplinary Health Sciences, Augusta University in Augusta, GA. 


\title{
1IJAHSP ${ }^{m}$ \\ The Internet Joumnal of Allied Health Sciences and Practice \\ Dedicated to allied health professional practice and education \\ Vol. 20 No. 1 ISSN 1540-580X
}

\section{Attitudes and Beliefs of Physical Therapist and Physical Therapist Assistant Program Directors in the United States Towards Interprofessional Education}

\author{
Lawrence Ramiscal \\ Christopher Truelove Jr. \\ Vahe Heboyan \\ Gianluca De Leo \\ Augusta University \\ United States
}

\begin{abstract}
Purpose: To investigate the attitudes and beliefs of physical therapy (PT) and physical therapist assistant (PTA) program directors towards interprofessional education (IPE). We hypothesized that Communication and Ethics would be the most important competencies among program directors. Methods: A cross-sectional survey based on previously utilized instruments modified for the profession of PT was sent to PT and PTA program directors. One hundred sixteen responses were analyzed using frequency analysis for demographic data and non-parametric Mann-Whitney $U$ t-test for group differences. Results: While the majority of program directors agree that IPE is important, with Communication as the most important IPE competency, most PTA program directors do not support the importance of accreditation in implementing IPE $(p<0.05)$, unable to accommodate it in their current curricula $(p<0.05)$ and believe lack of support from Administration $(p<0.05)$. Although the majority of PT program directors believe they have the resources to implement IPE $(p<0.05)$, they disagree that IPE better utilizes resources $(p<0.05)$. Conclusion: Program directors agree that IPE is vital to student learning, with Communication as the most important IPE competency. However, specific differences between PT and PTA program directors emerged primarily on IPE implementation, the role of accreditation, resource support, and resource utilization.
\end{abstract}

Keywords: competencies, interprofessional education, physical therapy, survey 


\section{INTRODUCTION}

Widespread patient error in U.S. healthcare has revealed the inadequacies of costly care delivery systems. ${ }^{1}$ It shows that how care is delivered is as important as what care is delivered. ${ }^{2}$ Optimal healthcare delivery to patients extends beyond what was provided by a single health profession and requires collaboration by multiple healthcare providers. ${ }^{3}$ The recent increase of interdisciplinary teams in healthcare settings has made clear that interprofessional education (IPE) effort is required to prepare future healthcare students to function more effectively in this continuously evolving environment. There is growing evidence showing that IPE implementation across healthcare curricula can positively impact students' attitudes, beliefs, knowledge, skills, and collaborative competencies on IPE. Ultimately, IPE can improve professional practice and clinical outcomes. ${ }^{4}$

The World Health Organization defines IPE as "occasions in which two or more professions learn, with, from and about each other to improve collaboration and quality of care."3 In 2009, dentistry, medicine, nursing, pharmacy, and public health formed the Interprofessional Education Collaborative (IPEC) to help advance IPE and promote team-based care. The IPEC expert panel defined four core competency domains: 1) Values/Ethics for Interprofessional Practice; 2) Roles/Responsibilities; 3) Interprofessional Communication; and 4) Teamwork. ${ }^{3}$ The purpose was to help guide the development of health professions curricula and prepare students to practice teamwork and team-based health care effectively. Since then, several health professions have adopted the core competencies in their IPE curriculum, including physical therapy (PT) education. 5,6

Over a decade later, many health professions have evaluated IPE in their respective educational programs, particularly the attitudes and beliefs towards IPE, including the IPEC core competencies, based on characteristics unique to their respective programs. ${ }^{7-9}$ According to these studies, IPE is recognized as an essential component of education across the board despite faculty's varying attitudes and beliefs regarding IPE amongst these professions. However, to the best of our knowledge, such evaluation has yet to be investigated in PT education. Therefore, the purpose of this study was to investigate the current attitudes and beliefs of PT and physical therapist assistant (PTA) program directors towards IPE. We hypothesized that IPE would be important, with Communication and Ethics as the most important competencies among program directors. We also hypothesized differences between PT and PTA program directors based on resources, implementation, and accreditation despite an intraprofessional (within the profession) relationship.

\section{METHODS}

\section{Study Design}

We utilized a cross-sectional survey design using a single-stage anonymous questionnaire. This study was approved by the Institutional Review Board of Augusta University, IRB\# 1313607-1, and all participants provided electronic consent.

\section{Subjects}

The target population for this survey was PT and PTA program directors. We obtained a list of accredited PT and PTA programs within the United States and US territories from the American Physical Therapy Association (APTA) website in October 2018, which contained the e-mail addresses of program directors..$^{10} \mathrm{~A}$ total of 457 email addresses were identified.

\section{Materials}

We designed a 73-item survey based on previously utilized instruments and modified for the profession of Physical Therapy.8, 9, 11 We organized the survey into six sections: (I) program information; (II) respondent information; (III) IPEC Competencies; (IV) attitudes toward IPE; (V) attitudes and beliefs about interprofessional learning in the academic setting; and (VI) attitude toward interprofessional health care teams.

In section I, we asked about what type of program is being offered in their institution. Section II focused on respondent information such as highest academic degree, current academic rank, and years in academia. Section IIII asked respondents to rank the four IPEC competencies (Values/Ethics, Role/Responsibilities, Interprofessional Communication, and Teams and Teamwork) in the order of importance. Finally, we adapted sections IV, V, and VI on a 5-point Likert-scale survey created by Curran for Interprofessional Education's study utilized in other previous studies. ${ }^{9}$ The Curran survey response options were "strongly disagree" (1), "disagree" (2), "neither agree nor disagree" (3), "agree" (4) and "strongly agree" (5).

We utilized Qualtrics ${ }^{12}$ to develop and distribute the survey. We distributed the survey via email to all PT and PTA program directors (457 email addresses) of APTA accredited programs in the United States and Puerto Rico from January to February of 2019. We sent a pilot survey to 49 random PT/PTA program directors to help identify the invitation or survey problems. One week later, we distributed the survey to the remaining 408 PT/PTA program directors. Five emails were undeliverable. In all, 452 program directors received an invitation to complete our survey. Every two weeks, we sent a reminder for the six weeks the survey was open to increase the response rate. 
Only 124 (27.4\%) of the 452 program directors submitted responses. We omitted three responses for duplicate selection of program type (121 remaining). There were 14 incomplete responses. However, incomplete responses were included if the respondent answered through the first question of section III (ranking the IPEC competencies). Five incomplete responses were excluded from the analysis. In all, we analyzed 116 (25.7\%) program director responses (116 responses through section III; 110 responses through the Curran sections IV, V, VI).

\section{Data Analysis}

At the end of the survey period, we extracted the response data from Qualtrics and exported it to IBM SPSS ${ }^{13}$ for analysis. We performed frequency analysis on all demographic questions. We gathered survey data using a 5-point Likert scale ranging from "strongly disagree" to "strongly agree." We calculated the mean and used the non-parametric Mann-Whitney U t-test to determine differences in responses between the groups (PT vs. PTA). The significance level was set to 0.05 for all analyses.

Although the survey we used to gather data had a 5-point scale from the Curran survey (sections IV, V, VI), we decided to collapse the data to a 3-point scale during the analysis. The 5-point scale yielded very few statistical differences. We visually inspected the data and noticed that most group differences were between the scales of 1 and 2 or 4 and 5 . To capture meaningful differences between overall agreement, disagreement, and indifference among the respondents, we combined responses 1 and 2 and 4 and 5. The new 3-point scale options were (1) "disagree," (2) "neither agree nor disagree," and (3) "agree." Collapsing the data to dichotomous or trichotomous scales have shown to perform well when compared to the data collected using the original 5-point scale during analysis, suggesting that collapsed data could replace the original scale at least in the analysis phase.14, 15

\section{RESULTS}

Respondent and Program Demographics

Table 1 displays the demographic data for all respondents. Table 2 displays the program demographics.

Table 1. Respondent Demographics

\begin{tabular}{|c|c|c|c|c|c|c|}
\hline \multirow[b]{2}{*}{ Characteristic } & \multirow{2}{*}{\multicolumn{2}{|c|}{$\begin{array}{l}\text { Total } \\
(n=116)\end{array}$}} & \multicolumn{4}{|c|}{ Program Type } \\
\hline & & & \multicolumn{2}{|c|}{$\begin{array}{l}\text { PTA } \\
(n=63)\end{array}$} & \multicolumn{2}{|c|}{$\begin{array}{l}\text { PT } \\
(n=53)\end{array}$} \\
\hline \multicolumn{7}{|l|}{ Highest degree achieved } \\
\hline Associate's & 3 & $(2.6)$ & 3 & $(100.0)$ & 0 & $(0.0)$ \\
\hline Bachelor's & 1 & (0.9) & 1 & $(100.0)$ & 0 & $(0.0)$ \\
\hline Master's & 30 & $(25.9)$ & 29 & $(96.7)^{\prime}$ & 1 & (3.3) \\
\hline Doctorate & 82 & (70.7) & 30 & $(36.6)$ & 52 & $(63.4)$ \\
\hline Prefer not to answer & 0 & $(0.0)^{\prime}$ & 0 & $(0.0)$ & 0 & $(0.0)^{\prime}$ \\
\hline \multicolumn{7}{|l|}{ Academic rank } \\
\hline Instructor & 5 & $(4.3)$ & 5 & $(100.0)$ & 0 & $(0.0)$ \\
\hline Lecturer & 1 & $(0.9)$ & 1 & $(100.0)$ & 0 & $(0.0)$ \\
\hline Assistant Clinical & 5 & (4.3) & 2 & $(40.0)$ & 3 & $(60.0)$ \\
\hline Associate Clinical Professor & 8 & (6.9) & 0 & $(0.0)$ & 8 & $(100.0)$ \\
\hline Clinical Professor & 2 & $(1.7)$ & 0 & $(0.0)$ & 2 & $(100.0)$ \\
\hline Assistant Professor & 7 & $(6.0)$ & 5 & $(71.4)$ & 2 & $(28.6)$ \\
\hline Associate Professor & 29 & $(25.0)$ & 14 & $(48.3)$ & 15 & $(51.7)$ \\
\hline Professor & 42 & (36.2) & 19 & $(45.2)$ & 23 & $(54.8)$ \\
\hline Other & 15 & (12.9) & 15 & $(100.0)$ & 0 & $(0.0)$ \\
\hline Prefer not to answer & 2 & $(1.7)$ & 2 & $(100.0)$ & 0 & $(0.0)$ \\
\hline \multicolumn{7}{|l|}{ Years in academia } \\
\hline$<5$ years & 13 & $(11.2)$ & 11 & $(84.6)$ & 2 & $(15.4)$ \\
\hline $6-10$ years & 19 & (16.4) & 16 & $(84.2)$ & 3 & $(15.8)$ \\
\hline $11-15$ years & 26 & $(22.4)$ & 14 & $(53.8)$ & 12 & $(46.2)$ \\
\hline $16-20$ years & 18 & (15.5) & 9 & $(50.0)$ & 9 & $(50.0)$ \\
\hline$>20$ years & 40 & (34.5) & 13 & $(32.5)$ & 27 & $(67.5)$ \\
\hline Prefer not to answer & 0 & $(0.0)$ & 0 & $(0.0)$ & 0 & $(0.0)$ \\
\hline \multicolumn{7}{|c|}{ Agreement with WHO's definition of IPE } \\
\hline Yes & 112 & (96.6) & 60 & $(53.6)$ & 52 & $(46.4)$ \\
\hline
\end{tabular}

(C) The Internet Journal of Allied Health Sciences and Practice, 2022 


\begin{tabular}{lllllll} 
No & 3 & $(2.6)$ & 2 & $(66.7)$ & 1 & $(33.3)$ \\
Prefer not to answer & 1 & $(0.9)$ & 1 & $(100.0)$ & 0 & $(0.0)$ \\
Opinion on necessary credit hours to teach IPE & & & & \\
Zero & 16 & $(13.8)$ & 12 & $(75.0)$ & 4 & $(25.0)$ \\
One & 9 & $(7.8)$ & 7 & $(77.8)$ & 2 & $(22.2)$ \\
Two & 18 & $(15.5)$ & 10 & $(55.6)$ & 8 & $(44.4)$ \\
Three & 8 & $(6.9)$ & 3 & $(37.5)$ & 5 & $(62.5)$ \\
Four or more & 16 & $(13.8)$ & 5 & $(31.3)$ & 11 & $(68.8)$ \\
Do not know & 41 & $(35.3)$ & 24 & $(58.5)$ & 17 & $(41.5)$ \\
Prefer not to answer & 8 & $(6.9)$ & 2 & $(25.0)$ & 6 & $(75.0)$ \\
\hline
\end{tabular}

Note. Results are $\mathrm{n}(\%) ; \mathrm{PT}=$ physical therapy; PTA = physical therapist assistant; IPE = interprofessional education; WHO = World Health Organization

Table 2. Program Demographics

\begin{tabular}{|c|c|c|c|c|c|c|}
\hline \multirow[b]{2}{*}{ Characteristic } & \multirow{2}{*}{\multicolumn{2}{|c|}{$\begin{array}{l}\text { Total } \\
(n=116)\end{array}$}} & \multicolumn{4}{|c|}{ Program Type } \\
\hline & & & \multicolumn{2}{|c|}{$\begin{array}{l}\text { PTA } \\
(n=63)\end{array}$} & \multicolumn{2}{|c|}{$\begin{array}{l}\text { PT } \\
(n=53)\end{array}$} \\
\hline \multicolumn{7}{|l|}{ Private v. Public } \\
\hline Private & 46 & $(39.7)$ & 14 & $(30.4)$ & 32 & $(69.6)$ \\
\hline Public & 70 & (60.3) & 49 & $(70.0)$ & 21 & $(30.0)$ \\
\hline \multicolumn{7}{|l|}{ Traditional vs Hybrid } \\
\hline Traditional & 105 & $(90.5)$ & 60 & $(57.1)$ & 45 & $(42.9)$ \\
\hline Hybrid & 11 & (9.5) & 3 & (27.3) & 8 & $(72.7)$ \\
\hline \multicolumn{7}{|l|}{ Number of Students } \\
\hline$<50$ & 58 & $(50.0)$ & 49 & $(84.5)$ & 9 & $(15.5)$ \\
\hline $51-100$ & 23 & (19.8) & 13 & $(56.5)$ & 10 & $(43.5)$ \\
\hline $101-150$ & 16 & (13.8) & 1 & $(6.3)$ & 15 & (93.8) \\
\hline $151-200$ & 11 & $(9.5)^{\prime}$ & 0 & $(0.0)$ & 11 & $(100.0)$ \\
\hline $201-250$ & 4 & (3.4) & 0 & $(0.0)$ & 4 & (100.0) \\
\hline$>250$ & 3 & (2.6) & 0 & $(0.0)$ & 3 & $(100.0)$ \\
\hline Prefer not to answer & 1 & $(0.9)$ & 0 & $(0.0)$ & 1 & $(100.0)$ \\
\hline \multicolumn{7}{|l|}{ Program age } \\
\hline$<5$ years & 13 & $(11.2)$ & 7 & $(53.8)$ & 6 & $(46.2)$ \\
\hline $6-10$ years & 12 & (10.3) & 10 & (83.3) & 2 & (16.7) \\
\hline $11-15$ years & 5 & (4.3) & 4 & $(80.0)$ & 1 & $(20.0)$ \\
\hline $16-20$ years & 1 & $(0.9)$ & 1 & $(100.0)$ & 0 & $(0.0)$ \\
\hline$>20$ years & 84 & $(72.4)$ & 41 & $(48.8)$ & 43 & $(51.2)$ \\
\hline Prefer not to answer & 1 & $(0.9)$ & 0 & $(0.0)$ & 1 & $(100.0)$ \\
\hline
\end{tabular}

Note. Results are $\mathrm{n}(\%) ; \mathrm{PT}=$ physical therapy; PTA = physical therapist assistant

\section{IPEC Ranking}

Table 3 presents the respondent's ranking of the four IPEC competencies from least to most important. Based on the frequency count, the Figure shows that Communication was considered the most important competency amongst the respondents while Values/Ethics is the least important IPEC competency reported. A Mann-Whitney U non-parametric t-test showed no significant differences between the groups. 
Table 3. Comparison of the Interprofessional Education Collaborative Competencies Ranking Between the Groups (programs)

\begin{tabular}{|c|c|c|c|c|c|c|c|c|c|}
\hline $\begin{array}{l}\text { Competencies } \\
\text { Communication }\end{array}$ & \multicolumn{2}{|c|}{$\begin{array}{l}\text { Most } \\
\text { Important }\end{array}$} & \multicolumn{2}{|c|}{$\begin{array}{l}\text { More } \\
\text { Important }\end{array}$} & \multicolumn{2}{|c|}{ Somewhat Important } & \multicolumn{2}{|c|}{$\begin{array}{l}\text { Least } \\
\text { Important }\end{array}$} & $p$ \\
\hline Total $(n=116)$ & 42 & $(36.2)$ & 45 & $(38.8)$ & 21 & $(18.1)$ & 8 & $(6.9)$ & \multirow{3}{*}{0.124} \\
\hline $\operatorname{PTA}(n=63)$ & 22 & (34.9) & 20 & (31.7) & 13 & $(20.6)$ & 8 & $(12.7)$ & \\
\hline $\mathrm{PT}(\mathrm{n}=53)$ & 20 & $(37.7)$ & 25 & $(47.2)$ & 8 & $(15.1)$ & 0 & $(0.0)$ & \\
\hline \multicolumn{10}{|c|}{ Roles and Responsibilities } \\
\hline Total $(n=116)$ & 33 & $(28.4)$ & 36 & $(31.0)$ & 19 & $(16.4)$ & 28 & $(24.1)$ & \multirow{3}{*}{0.061} \\
\hline $\operatorname{PTA}(n=63)$ & 21 & (33.3) & 20 & (31.7) & 12 & $(19.0)$ & 10 & (15.9) & \\
\hline PT $(n=53)$ & 12 & (22.6) & 16 & $(30.2)$ & 7 & (13.2) & 18 & $(34.0)$ & \\
\hline \multicolumn{10}{|l|}{ Values and Ethics } \\
\hline Total $(n=116)$ & 27 & $(23.3)$ & 11 & (9.5) & 30 & $(25.9)$ & 48 & $(41.4)$ & \multirow{3}{*}{0.808} \\
\hline PTA $(n=63)$ & 14 & $(22.2)$ & 7 & (11.1) & 15 & $(23.8)$ & 27 & $(42.9)$ & \\
\hline $\mathrm{PT}(\mathrm{n}=53)$ & 13 & (24.5) & 4 & $(7.5)$ & 15 & $(28.3)$ & 21 & $(39.6)$ & \\
\hline \multicolumn{10}{|c|}{ Teams and Teamwork } \\
\hline Total $(n=116)$ & 14 & $(12.1)$ & 24 & (20.7) & 46 & $(39.7)$ & 32 & $(27.6)$ & \multirow{3}{*}{0.967} \\
\hline PTA $(n=63)$ & 6 & $(9.5)$ & 16 & $(25.4)$ & 23 & $(36.5)$ & 18 & $(28.6)$ & \\
\hline $\mathrm{PT}(n=53)$ & 8 & (15.1) & 8 & (15.1) & 23 & $(43.4)$ & 14 & $(26.4)$ & \\
\hline
\end{tabular}

Note. Results are $\mathrm{n}(\%) ; p=0.05 ; \mathrm{PT}=$ physical therapy; PTA = physical therapist assistant

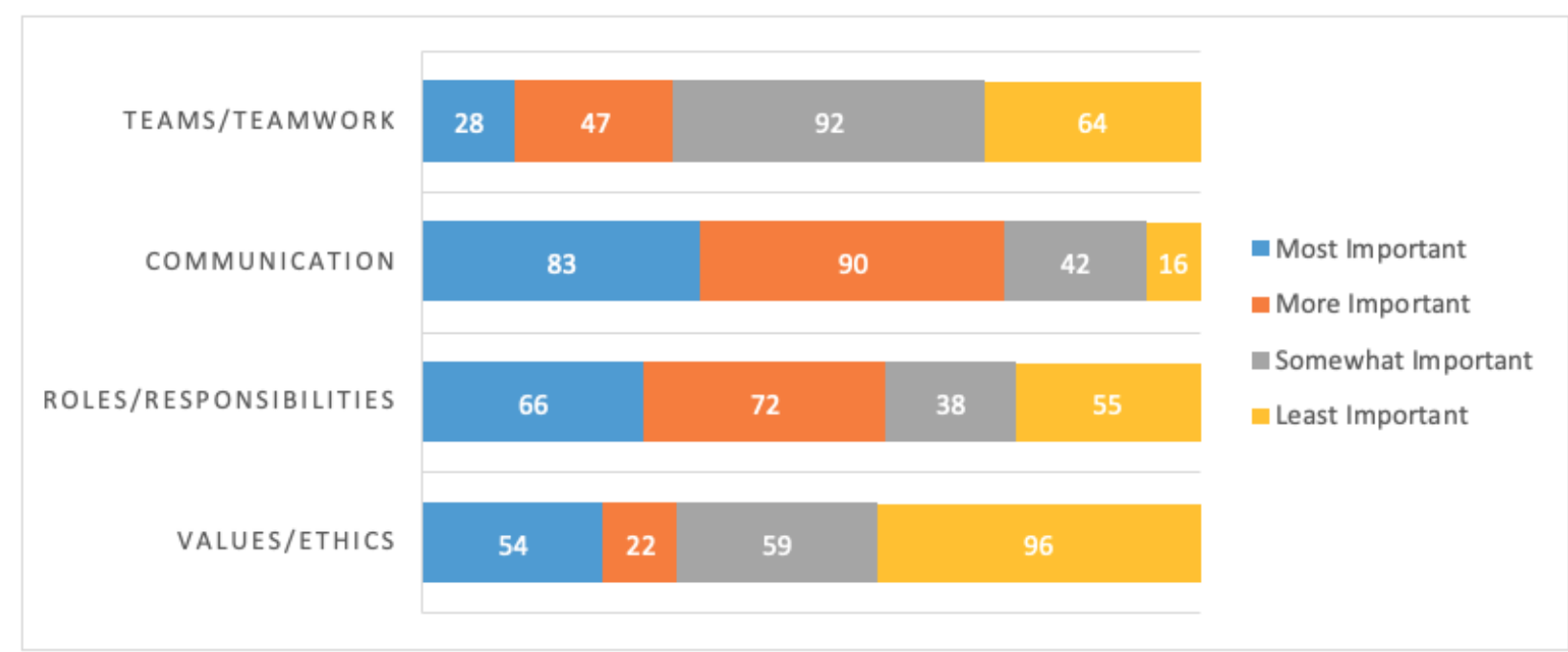

Figure. Ranking of the Importance of the Interprofessional Education Collaborative Competencies by all Program Directors

\section{Attitudes and Beliefs toward IPE}

Table 4 summarizes the results of the survey on attitudes toward IPE (Curran survey: sections IV, V, VI) and corresponding items that showed significant differences between the groups of interest using the Mann-Whitney $U$ non-parametric t-test. 
Table 4. Attitudes Towards IPE (Curran) Likert Scale Questions, Responses, and Differences Between Groups (programs)

\begin{tabular}{|c|c|c|c|c|c|c|c|c|c|}
\hline \multirow[b]{2}{*}{ Questions } & \multirow{2}{*}{\begin{tabular}{l}
\multicolumn{1}{c}{ Program } \\
Total $(n=110)$ \\
PT $(n=50)$ \\
PTA $(n=60)$
\end{tabular}} & \multicolumn{7}{|c|}{ Response } & \multirow[b]{2}{*}{$p$} \\
\hline & & \multicolumn{2}{|c|}{ Disagree (1) } & \multicolumn{2}{|c|}{$\begin{array}{l}\text { Neither } \\
\text { Agree nor } \\
\text { Disagree (2) }\end{array}$} & \multicolumn{2}{|c|}{ Agree (3) } & Mean \pm SD & \\
\hline \multicolumn{9}{|l|}{ Attitudes toward interprofessional education } & \multirow{3}{*}{0.184} \\
\hline \multirow{2}{*}{$\begin{array}{l}\text { Interprofessional learning will help } \\
\text { students think positively about } \\
\text { other health care professionals. }\end{array}$} & PT & 0 & (0) & 6 & $(12)$ & 44 & $(88)$ & $2.88 \pm 0.33$ & \\
\hline & PTA & 0 & $(0)$ & 3 & (5) & 57 & (95) & $2.95 \pm 0.22$ & \\
\hline \multirow{2}{*}{$\begin{array}{l}\text { Clinical problem solving can only } \\
\text { be learned effectively when } \\
\text { students are taught within their } \\
\text { individual department/school. }\end{array}$} & PT & 44 & (88) & 3 & (6) & 3 & $(6)$ & $1.18 \pm 0.52$ & \multirow{2}{*}{0.300} \\
\hline & PTA & 48 & $(80)$ & 9 & $(15)$ & 3 & $(5)$ & $1.25 \pm 0.54$ & \\
\hline \multirow{2}{*}{$\begin{array}{l}\text { Patients would ultimately benefit if } \\
\text { health care students worked } \\
\text { together to solve patient problems. }\end{array}$} & PT & 0 & (0) & 0 & (0) & 50 & $(100)$ & $3.00 \pm 0.00$ & \multirow{2}{*}{0.064} \\
\hline & PTA & 0 & (0) & 4 & (7) & 56 & (93) & $2.93 \pm 0.25$ & \\
\hline \multirow{2}{*}{$\begin{array}{l}\text { Students in physical therapy would } \\
\text { benefit from working on small- } \\
\text { group projects with other health } \\
\text { care students. }\end{array}$} & PT & 1 & (2) & 2 & (4) & 47 & (94) & $2.92 \pm 0.34$ & \multirow{2}{*}{0.665} \\
\hline & PTA & 0 & (0) & 5 & (8) & 55 & (92) & $2.92 \pm 0.30$ & \\
\hline \multirow{2}{*}{$\begin{array}{l}\text { Communication skills should be } \\
\text { learned with integrated classes of } \\
\text { health care students. }\end{array}$} & PT & 1 & (2) & 13 & $(26)$ & 36 & (72) & $2.70 \pm 0.51$ & \multirow{2}{*}{0.240} \\
\hline & PTA & 6 & (10) & 16 & $(27)$ & 38 & (63) & $2.53 \pm 0.68$ & \\
\hline \multirow{4}{*}{$\begin{array}{l}\text { Learning with students in other } \\
\text { health professional schools helps } \\
\text { students to become more effective } \\
\text { members of a health care team. } \\
\text { Interprofessional learning among } \\
\text { health care students will increase } \\
\text { their ability to understand clinical } \\
\text { problems. }\end{array}$} & PT & 0 & (0) & 4 & (8) & 46 & (92) & $2.92 \pm 0.27$ & \multirow{3}{*}{0.790} \\
\hline & PTA & 0 & (0) & 4 & (7) & 56 & (93) & $2.93 \pm 0.25$ & \\
\hline & PT & 2 & (4) & 7 & (14) & 41 & (82) & $2.78 \pm 0.51$ & \\
\hline & PTA & 1 & (2) & 12 & $(20)$ & 47 & (78) & $2.77 \pm 0.46$ & 0.688 \\
\hline \multirow{2}{*}{$\begin{array}{l}\text { Interprofessional learning will help } \\
\text { students to understand their own } \\
\text { professional limitations. }\end{array}$} & PT & 4 & (8) & 3 & (6) & 43 & (86) & $2.78 \pm 0.58$ & \multirow{2}{*}{0.310} \\
\hline & PTA & 1 & (2) & 13 & $(22)$ & 46 & (77) & $2.75 \pm 0.47$ & \\
\hline \multirow{2}{*}{$\begin{array}{l}\text { For small-group learning to work, } \\
\text { students need to trust and respect } \\
\text { each other. }\end{array}$} & PT & 0 & (0) & 3 & (6) & 47 & (94) & $2.94 \pm 0.24$ & \multirow{2}{*}{0.870} \\
\hline & PTA & 1 & (2) & 3 & (5) & 56 & (93) & $2.92 \pm 0.33$ & \\
\hline \multicolumn{9}{|c|}{ Attitudes and beliefs about interprofessional learning in the academic setting } & \multirow{3}{*}{0.000} \\
\hline \multirow{2}{*}{$\begin{array}{l}\text { There are current curriculum } \\
\text { requirements that could be } \\
\text { removed to make room for } \\
\text { additional IPE education }\end{array}$} & PT & 18 & (36) & 12 & $(24)$ & 20 & $(40)$ & $2.04 \pm 0.88$ & \\
\hline & PTA & 41 & (69) & 10 & $(17)$ & 8 & $(14)$ & $1.44 \pm 0.73$ & \\
\hline \multirow{2}{*}{$\begin{array}{l}\text { CAPTE should mandate IPE in the } \\
\text { PT/PTA curriculum }\end{array}$} & PT & 10 & (20) & 5 & $(10)$ & 35 & $(70)$ & $2.50 \pm 0.81$ & 00000 \\
\hline & PTA & 23 & (39) & 19 & $(32)$ & 17 & $(29)$ & $1.90 \pm 0.82$ & 0.000 \\
\hline My program has the resources and & PT & 4 & (8) & 12 & $(24)$ & 34 & $(68)$ & $2.60 \pm 0.64$ & 0000 \\
\hline personnel to teach IPE courses & PTA & 30 & (51) & 12 & $(20)$ & 17 & (29) & $1.78 \pm 0.87$ & 0.000 \\
\hline My institution has the resources to & PT & 8 & (16) & 10 & $(20)$ & 32 & $(64)$ & $2.48 \pm 0.76$ & 36 \\
\hline implement IPE & PTA & 16 & (27) & 9 & $(15)$ & 34 & $(57)$ & $2.31 \pm 0.88$ & \\
\hline & PT & 13 & (26) & 21 & $(42)$ & 16 & (32) & $2.06 \pm 0.77$ & 0.042 \\
\hline
\end{tabular}




\begin{tabular}{|c|c|c|c|c|c|c|c|c|c|}
\hline $\begin{array}{l}\text { Interprofessional learning better } \\
\text { utilizes resources }\end{array}$ & PTA & 6 & $(10)$ & 26 & (44) & 27 & (46) & $2.36 \pm 0.66$ & \multirow{3}{*}{0.282} \\
\hline \multirow{2}{*}{$\begin{array}{l}\text { Faculty should be encouraged to } \\
\text { participate in interprofessional } \\
\text { courses }\end{array}$} & $\mathrm{PT}$ & 0 & (0) & 12 & (24) & 38 & (76) & $2.76 \pm 0.43$ & \\
\hline & PTA & 3 & (5) & 16 & (27) & 40 & (68) & $2.63 \pm 0.58$ & \\
\hline \multirow{2}{*}{$\begin{array}{l}\text { Faculty like teaching with faculty } \\
\text { from other academic departments }\end{array}$} & PT & 5 & $(10)$ & 19 & (38) & 26 & (52) & $2.42 \pm 0.67$ & \multirow{2}{*}{0.621} \\
\hline & PTA & 7 & (12) & 24 & (41) & 28 & (47) & $2.36 \pm 0.69$ & \\
\hline \multirow{2}{*}{$\begin{array}{l}\text { Interprofessional efforts weaken } \\
\text { program content }\end{array}$} & PT & 44 & $(88)$ & 6 & (12) & 0 & $(0)$ & $1.12 \pm 0.33$ & \multirow{2}{*}{0.203} \\
\hline & PTA & 47 & $(80)$ & 8 & (13) & 4 & (7) & $1.27 \pm 0.58$ & \\
\hline \multirow{2}{*}{$\begin{array}{l}\text { Interprofessional efforts require } \\
\text { support from campus } \\
\text { administration }\end{array}$} & PT & 1 & (2) & 1 & (2) & 48 & (96) & $2.94 \pm 0.31$ & \multirow[b]{2}{*}{0.141} \\
\hline & PTA & 3 & (5) & 4 & (7) & 52 & (88) & $2.83 \pm 0.50$ & \\
\hline \multirow{2}{*}{$\begin{array}{l}\text { Interprofessional courses are } \\
\text { logistically difficult }\end{array}$} & PT & 2 & (4) & 3 & (6) & 45 & (90) & $2.86 \pm 0.45$ & \multirow{2}{*}{0.321} \\
\hline & PTA & 2 & (3) & 8 & (14) & 49 & (83) & $2.80 \pm 0.48$ & \\
\hline \multirow{2}{*}{$\begin{array}{l}\text { Accreditation requirements limit } \\
\text { interprofessional efforts }\end{array}$} & PT & 31 & $(62)$ & 14 & (28) & 5 & (10) & $1.48 \pm 0.68$ & \multirow{2}{*}{0.000} \\
\hline & PTA & 16 & $(27)$ & 22 & (37) & 21 & (36) & $2.08 \pm 0.79$ & \\
\hline \multirow{2}{*}{$\begin{array}{l}\text { It is important for academic health } \\
\text { center campuses to provide } \\
\text { interprofessional learning } \\
\text { opportunities }\end{array}$} & PT & 0 & (0) & 10 & (20) & 40 & (80) & $2.80 \pm 0.40$ & \multirow{2}{*}{0.186} \\
\hline & PTA & 2 & (3) & 16 & (27) & 41 & (70) & $2.66 \pm 0.55$ & \\
\hline \multicolumn{9}{|c|}{ Attitudes toward interprofessionalism in health care teams } & \multirow{4}{*}{0.767} \\
\hline \multirow{2}{*}{$\begin{array}{l}\text { Patients/clients receiving } \\
\text { interprofessional care are more } \\
\text { likely than others to be treated as } \\
\text { whole persons }\end{array}$} & PT & 4 & (8) & 11 & (22) & 35 & $(70)$ & $2.62 \pm 0.64$ & \\
\hline & PTA & 8 & $(14)$ & 10 & (17) & 40 & $(53)$ & $2.22 \pm 0.73$ & \\
\hline \multirow{2}{*}{$\begin{array}{l}\text { The give and take among team } \\
\text { members help them make better } \\
\text { patient/client decisions }\end{array}$} & PT & 3 & (2) & 6 & (12) & 41 & (82) & $2.76 \pm 0.56$ & \\
\hline & PTA & 1 & $(2)$ & 9 & (15) & 48 & (83) & $2.81 \pm 0.44$ & 0.841 \\
\hline \multirow{4}{*}{$\begin{array}{l}\text { The interprofessional approach } \\
\text { makes the delivery of care more } \\
\text { efficient } \\
\text { Working in an interprofessional } \\
\text { environment keeps most health } \\
\text { professionals enthusiastic and } \\
\text { interested in their jobs }\end{array}$} & PT & 2 & (4) & 19 & (38) & 29 & $(58)$ & $2.54 \pm 0.58$ & \multirow[b]{2}{*}{0.012} \\
\hline & PTA & 2 & (3) & 9 & (16) & 47 & (81) & $2.78 \pm 0.50$ & \\
\hline & PT & 0 & $(0)$ & 25 & (50) & 25 & $(50)$ & $2.55 \pm 0.51$ & \multirow{2}{*}{0.376} \\
\hline & PTA & 4 & (7) & 18 & (31) & 36 & (62) & $2.31 \pm 0.63$ & \\
\hline \multirow{2}{*}{$\begin{array}{l}\text { The interprofessional approach } \\
\text { improves the quality of care to } \\
\text { patient/clients }\end{array}$} & PT & 0 & $(0.0)$ & 7 & (14) & 43 & (86) & $2.86 \pm 0.35$ & \multirow{2}{*}{0.562} \\
\hline & PTA & 0 & $(0.0)$ & 6 & (10) & 52 & (90) & $2.90 \pm 0.31$ & \\
\hline \multirow{4}{*}{$\begin{array}{l}\text { Having to report observations to a } \\
\text { team helps team members to } \\
\text { better understand the work of other } \\
\text { health professionals } \\
\text { Team meetings foster } \\
\text { communication among members } \\
\text { from different professional or } \\
\text { disciplines }\end{array}$} & PT & 1 & (2) & 3 & (6) & 46 & (92) & $2.90 \pm 0.36$ & \multirow{2}{*}{0.933} \\
\hline & PTA & 0 & $(0.0)$ & 5 & (8) & 53 & (92) & $2.91 \pm 0.28$ & \\
\hline & PT & 0 & $(0.0)$ & 1 & (2) & 49 & (98) & $2.98 \pm 0.14$ & \multirow{2}{*}{0.134} \\
\hline & PTA & 1 & $(2)$ & 4 & (7) & 53 & (91) & $2.90 \pm 0.36$ & \\
\hline \multirow{2}{*}{$\begin{array}{l}\text { Working in an interprofessional } \\
\text { manner unnecessarily complicates } \\
\text { things most of the time }\end{array}$} & PT & 36 & (72) & 10 & (20) & 4 & (8) & $1.36 \pm 0.63$ & 0.368 \\
\hline & PTA & 46 & (79) & 9 & (16) & 3 & (5) & $1.26 \pm 0.55$ & \\
\hline
\end{tabular}

Note. Results are $\mathrm{n}(\%) ; \mathrm{SD}=$ standard deviation; significant results are in bold; $p=0.05 ; \mathrm{PT}=$ physical therapy; PTA = physical therapist assistant; IPE = interprofessional education; CAPTE = Commission on Accreditation in Physical Therapy Education 


\section{DISCUSSION}

We found that the majority of program directors identified that the most important IPE competency is Communication. Other health disciplines like Occupational Therapy (OT), Respiratory Therapy (RT), and Nutrition that investigated the importance of the IPEC competencies in their respective professional curriculum also found that Communication is the most important competency. ${ }^{7-9}$ Since Communication relates to effective interactions to support a team approach, according to these studies, this reflects how valuable it is for program directors that students learn to connect to the entire healthcare team, including choosing effective communication tools, expressing own knowledge and opinions, actively listening, providing feedback, and practicing respectful conversations with team members., ${ }^{3,-9}$ Although Values and Ethics were ranked last, each IPE competency is presumed important.

When it came to IPE specific to the academic setting, PTA program directors strongly feel that everything in their current curriculum is essential. Therefore, although the PTA program directors feel IPE is important, nothing else can be removed to give way to IPE activities. In the investigation among RT program directors, a similar response was observed. ${ }^{9}$ This response may be in part due to the length of the programs. Like most RT programs, PTA programs are only taught in two years as an associate degree, compared to the Doctor of Physical Therapy degree awarded by PT programs that can go to as long as seven years (including a four-year undergraduate Bachelor's degree). Currently, there is an ongoing debate regarding changing the PTA program from an Associate's to a Bachelor's degree. ${ }^{16}$ If the PTA program is lengthened, it may encourage its directors to include more IPE activities in their curriculum.

Most PT program directors support IPE being a requirement for accreditation, while the majority of the PTA program directors are not. Therefore, it was no surprise that those who disagreed on the follow-up item that accreditation requirements limit interprofessional efforts were mostly PT program directors. This response may imply that PT program directors believe mandating IPE for accreditation will help encourage developing more IPE activities into the PT education since IPE is mandatory in both didactic and clinical curriculum for PT accreditation, as opposed to PTA's requirements only for clinical education.5, 6 PT program directors also felt that their administration supports their program's IPE effort by providing resources to implement it, while PTA directors did not. This response may also be partly due to PT programs' length and accreditation requirements requiring the administration to support IPE activities. This corroborates the findings of Wise and colleagues with institutional support as one of the highest voted reasons for IPE success along with faculty buy-in in physical therapy programs. ${ }^{11}$

Most PT program directors disagreed that IPE better utilizes resources even though they state that they have the resources to implement IPE. It is possible that although support is already present through the availability of resources, it may not mean PT programs are utilizing these resources efficiently when implementing IPE activities. Although the study by Wise and colleagues surveyed PT programs four years earlier, our findings remain similar to theirs. They identified that poor organization and lack of schedule coordination were the biggest challenge to IPE implementation. ${ }^{11}$ The Guidance on Developing Quality Interprofessional Education for the Health Professions (Guidance) was recently published. ${ }^{17} \mathrm{Here}$, program-specific leaders, particularly Department Chairs and Program Directors, were identified as the primary stake-holder in creating and implementing IPE plans. The Guidance should be used by program directors, especially in PT, to improve its efficiency in utilizing resources for the development and effective implementation of quality IPE activities.

The majority of the program directors agreed with the importance of belonging to academic health center campuses to provide interprofessional learning opportunities. This seems consistent with the findings in other health disciplines like OT and RT as programs affiliated with healthcare systems have extensive access to additional shared institutional resources, thus enabling more access to IPE implementation opportunities.8,9

On attitudes towards interprofessionalism in health care teams, there was widespread agreement that the interprofessional approach makes care delivery efficient. The majority of those who agreed were PTA program directors. In contrast, more than onethird of PT program directors were still unsure. This may be explained as PTAs remain to function only under the supervision of PTs. Therefore, they must work alongside PTs all the time, while PTs may work independently due to direct access laws. This arrangement (PTAs are working under PTs) is technically labeled as intraprofessional (within the profession) versus interprofessional (with other professions). ${ }^{18}$

\section{Limitations}

There are a few limitations we identified in our study, starting with the response rate. Despite our best efforts to encourage responses, our rate was just under $28 \%$. This could be because of the overall length of the survey. However, a return rate of about $30 \%-60 \%$ is deemed realistic, therefore acceptable. ${ }^{19}$ Another limitation was the implementation timing in relation to the release of the Guidance. It is possible that program directors, after reading the Guidance, may provide a different response to the survey.

( The Internet Journal of Allied Health Sciences and Practice, 2022 


\section{Recommendations for Future Studies}

Future studies could assess the opinion of PT students on the importance of IPE education and their experience with IPE learning opportunities like it has been investigated among medical students. ${ }^{20}$ Other disciplines that rely on intraprofessional relationships (physicians and physician assistants, occupational therapists and certified occupational therapy assistants) may similarly explore IPE education to provide insight within their profession.

\section{CONCLUSION}

Program directors of PT and PTA programs agree that IPE is an important and integral part of student learning to produce successful healthcare practitioners and team members. This study suggests that the most important IPE competency their students need to achieve is Communication for most PT and PTA programs directors. However, specific differences between PT and PTA program directors emerged. Because PTA programs only offer an associate's degree, PTA program directors are generally hesitant in implementing IPE in their curricula. With IPE being limited as a requirement in PTA clinical curriculum only, most PTA program directors disagree with the importance of accreditation in implementing IPE in the entire curricula. Although PT programs directors believe they have more resources in implementing IPE due to accreditation requirements and relatively longer program length, they also believe that having IPE may not necessarily utilize these available resources efficiently. The recent Guidance may help programs become efficient in using resources, therefore effectively implementing IPE activities.

\section{REFERENCES}

1. Kohn LT, Corrigan J, Donaldson MS. To err is human : building a safer health system. National Academy Press; 2000:xxi, $287 \mathrm{p}$.

2. Core competencies for interprofessional collaborative practice: Report of an expert panel. Interprofessional Education Collaborative Expert Panel; 2011.

3. Core competencies for interprofessional collaborative practice: 2016 update. Washington, DC: Interprofessional Education Collaborative. 2016:1-19.

4. Reeves S, Fletcher $\mathrm{S}$, Barr $\mathrm{H}$, et al. A BEME systematic review of the effects of interprofessional education: BEME Guide No. 39. Med Teach. 2016;38(7):656-668.

5. Standards and Required Elements for Accreditation of Physical Therapist Education Programs. Commission on Accreditation in Physical Therapy Education. Accessed November 18, 2018.

http://www.capteonline.org/uploadedFiles/CAPTEorg/About_CAPTE/Resources/Accreditation_Handbook/CAPTE_PTS tandardsEvidence.pdf

6. Standards and Required Elements for Accreditation of Physical Therapist Assistant Education Programs. Commission on Accreditation in Physical Therapy Education. Accessed November 18, 2018. http://www.capteonline.org/uploadedFiles/CAPTEorg/About_CAPTE/Resources/Accreditation_Handbook/CAPTE_PTA StandardsEvidence.pdf

7. Patton Z, Vernon M, Haymond K, Anglin J, Heboyan V, De Leo G. Evaluation of Interprofessional Education Implementation among Nutrition Program Directors in the United States. Top Clin Nutr. 2018;33(3):196-204.

8. Hughes J, Allen A, McLane T, Stewart J, Heboyan V, De Leo G. Interprofessional Education among Occupational Therapy programs: Faculty perceptions of challenges and opportunities. Am J Occup Ther. 2018;

9. Vernon MM, Moore NM, Cummins LA, et al. Respiratory Therapy Faculty Knowledge of and Attitudes Toward Interprofessional Education. Respir Care. Jul 2017;62(7):873-881. doi:10.4187/respcare.05034

10. Accredited PT \& PTA Programs Directory. Commission on Accreditation in Physical Therapy Education American Physical Therapy Association CAPTE. Accessed October 31, 2018. http://aptaapps.apta.org/accreditedschoolsdirectory/default.aspx? UniqueKey=

11. Wise H, Frost J, Resnik C, Davis B, Inglarsh Z. Interprofessional Education: An Exploration in Physical Therapist Education. Journal of Physical Therapy Education. 2015;29(2)

12. Qualtrics. Version 1. 2019.

13. IBM SPSS Statistics for Mac. Version 25. 2019.

14. Jeong H, Lee W. The level of collapse we are allowed: Comparison of different response scales in Safety Attitudes Questionnaire. Biom Biostat Int J. 2016;4(4):00100.

15. Shearman D, Petocz P. When" Strongly Disagree" Doesn't Mean Strongly Disagree. Australian Association for Research in Education (NJ1). 2012;

16. Feasibility Study for Transitioning to an Entry-Level Baccalaureate Physical Therapist Assistant Degree. American Physical Therapy Association; 2014:

17. Guidance on developing quality interprofessional education for the health professions. Health Professions Accreditors Collaborative, Chicago, IL; 2019. 
18. Mahler C, Gutmann T, Karstens S, Joos S. Terminology for interprofessional collaboration: definition and current practice. GMS Zeitschrift für medizinische Ausbildung. 2014;31(4)

19. Watkins MP, Portney L. Foundations of clinical research: applications to practice. Pearson/Prentice Hall Upper Saddle River, NJ; 2009.

20. Zechariah S, Ansa BE, Johnson SW, Gates AM, De Leo G. Interprofessional Education and Collaboration in Healthcare: An Exploratory Study of the Perspectives of Medical Students in the United States. Multidisciplinary Digital Publishing Institute; 2019:117. 